\title{
Kinetic Model of a Carboxymethylcellulose-Agar Hydrogel for Long-Acting and Slow-Release of Chlorine Dioxide with a Modification of Fick's Diffusion Law
}

Lijie Huang, ${ }^{\text {a }}$ Hanyu Zhao, ${ }^{\text {a }}$ Hao Xu, ${ }^{\mathrm{a}}$ Minghui Qi, ${ }^{\mathrm{a}}$ Tan Yi, ${ }^{\mathrm{a}}$ Chongxing Huang, ${ }^{\mathrm{a}}$ Shuangfei Wang, ${ }^{\mathrm{b}, *}$ Shuxiang An, ${ }^{\mathrm{b}}$ and Chunying $\mathrm{Li}^{\mathrm{a}}$

A long-acting and slow-release material for chlorine dioxide, based on bagasse pulp (BP) was prepared with a superabsorbent resin as the slow-release substrate and agar as the cross-linking agent. The stable $\mathrm{ClO}_{2}$ solution and the acidic activator were locked into the network structure of the superabsorbent resin, which was prepared with a carboxymethyl cellulose made from bagasse pulp. Because of the network structure of the resin, the diffusion resistance was greatly increased, and the effective release time was up to 2 months. The mechanism for the release process of the $\mathrm{ClO}_{2}$ was explored, and a kinetic model was established based on modified Fick's diffusion law. The results showed that the release process was a diffusion-controlled process. When compared with a zero-order kinetic model and a Higuchi model, the new established model had better fitting results, and it more fully reflected the release patterns and characteristics of the $\mathrm{ClO}_{2}$.

Keywords: Fick's diffusion law; $\mathrm{ClO}_{2}$; Slow-release; Diffusion control; Kinetic mode

Contact information: a: Guangxi University, Nanning 530004, China; b: Guangxi Key Laboratory of Clean Pulp \& Papermaking and Pollution Control, Nanning 530004, China; *Corresponding authors: jiely165@163.com, wangsf@gxu.edu.cn

\section{INTRODUCTION}

$\mathrm{ClO}_{2}$ has been widely used in paper pulp and fiber bleaching, dealing with industrial wastewater, hospital sewage and industrial circulating cooling water, disinfecting and removing algae for poultry slaughter, indoor hygiene, beer manufacturing, oil exploration, food preservation, poultry and livestock production, aquaculture, and plant preservation (Hsu et al. 2015; Jiang et al. 2015; Fang et al. 2016 Han et al. 2017; Kaur et al. 2018). Chlorine dioxide aqueous solution is inconvenient for the preservation of fruits and vegetables during transportation and indoor air purification. Therefore, there has been a motivation to store and transport chlorine dioxide in an essentially solid form. The goal is to slowly releases a certain amount of $\mathrm{ClO}_{2}$ gas during an extended period after a special release agent is added (Ci et al. 2015; Long et al. 2016; Sun et al. $2017 \mathrm{a}, \mathrm{b}$ ). The starting time, effective release time, and release amount of adsorption type solid release system for $\mathrm{ClO}_{2}$ can be controlled, and it has broad applications in chemical and food industries.

It has been difficult to improve the stability and slow-release control of $\mathrm{ClO}_{2}$. There are different disadvantages for the slow-release $\mathrm{ClO}_{2}$ products currently used in the market, such as low $\mathrm{ClO}_{2}$ content, high release rates, poor control of the flow rates, a short effective working life, or the production of secondary pollution and waste resources. Therefore, it is important to produce $\mathrm{ClO}_{2}$ solution on the carrier via a release control technique to make a long-acting and slow-release $\mathrm{ClO}_{2}$ based on a solid material. To obtain an ideal release control effect, a special base material (usually a polymer material) is used to make an active agent release into the surroundings at a certain speed over a longer time. 
In this study, a solid material intended for the slow release of $\mathrm{ClO}_{2}$ (BP-SCD), was produced using a superabsorbent resin as a substrate and agar as a cross-linking agent, which connects an acidic active agent. The superabsorbent resin was made from bagasse pulp carboxymethyl cellulose and directly grafted with acroleic acid that was processed through low temperature plasma technology. This bypasses the need for N,N-methylene bisacrylamide, a crosslinking agent, and makes the resin easily degraded. It also achieved the integrative balance control between water and a variety of ingredients, as well as enabling the disinfection and sterilization efficacy of the long-acting and slow-releasing system for $\mathrm{ClO}_{2}$. The preparation process is simple, low cost, and easy to production. This paper explores the slow-release mechanism for $\mathrm{ClO}_{2}$ and established a kinetic model based on a modified Fick's diffusion law. The newly established model had better fitting results, and it can more fully reflect the releasing patterns and characteristics of the release system for chlorine dioxide, which can provided theoretical guidance for the quantitative research of release characteristics and the application of the products.

\section{EXPERIMENTAL}

\section{Materials}

Carboxymethyl cellulose was made via bagasse pulp etherification (BP-CMC) from a paper research institute (Guangxi University, Nanning, Guangxi, China). It was treated via an HPD atmospheric glow discharge and low-temperature plasma system (Nanjing Suman Electronics Limited Company, Nanjing, China) to produce a superabsorbent resin substrate (BP-SAR). Acrylic acid, sodium hydroxide, and potassium persulphate were all analytical reagents and were all purchased from Chengdu Kelon Chemical Reagent Factory (Chengdu, China), and the nitrogen was purchased from Guangxi Ruida Chemical Technology Co., Ltd. (Nanning, China) with a purity of $99.2 \%$.

\section{Methods}

\section{Preparation process of superabsorbent resin}

For the low-temperature plasma treatment, $2 \mathrm{~g}$ of dry BP-CMC samples were added into a clean watch glass and placed into the process chamber of the low-temperature plasma equipment, which was set to a certain vacuum degree $(300 \mathrm{~Pa})$, power $(250 \mathrm{~W})$, and radiation time $(90 \mathrm{~s})$. The vacuum valve was opened, allowing discharge to begin, and when the discharge was completed the samples were taken out immediately for the grafting reaction. For the graft polymerization, the BP-CMC that was treated in step one was placed into a conical flask with $50 \mathrm{~mL}$ of deionized water. The water temperature was set at $60^{\circ} \mathrm{C}$, ventilated with nitrogen, and then $\mathrm{NaOH}(45 \mathrm{~g})$, $\mathrm{C}_{3} \mathrm{H}_{4} \mathrm{O}_{2}\left(18 \mathrm{~g}\right.$ ), and $\mathrm{K}_{2} \mathrm{~S}_{2} \mathrm{O}_{8}$ (initiator, $0.5 \mathrm{~g}$ ) were added. The reaction was ended when the reaction liquid turned into a pink sticky liquid. A brown superabsorbent resin (BP-SAR) was formed after the pink liquid was vacuum-dried in at $60^{\circ} \mathrm{C}$.

\section{Preparation of solid release system for $\mathrm{ClO}_{2}$}

First, $2 \mathrm{~g}$ of BP-SAR was placed into $30 \mathrm{~mL}$ of $1 \% \mathrm{w} / \mathrm{w}$ stable $\mathrm{ClO}_{2}$ solution(sodium chlorite aqueous solution. After it was fully swollen, this "solution A" contained $309.8 \mathrm{mg}$ of effective $\mathrm{ClO}_{2}\left(G_{0}\right)$. Next, $4 \mathrm{~g}$ of agar was added into $30 \mathrm{~mL}$ of water, heated to boil, and mixed with $0.2 \mathrm{~g}$ of citric acid, which was called "solution B" after it cooled to approximately $45^{\circ} \mathrm{C}$. Solution A was quickly poured into B, stirred evenly, and cooled until coagulation occurred, which formed the release system for $\mathrm{ClO}_{2}$. Figure $\mathrm{S} 1$, in the appendix, shows the self-made slow-releasing solid chlorine dioxide, and the appearance is white or yellowish translucent solid; the device for 
preparing high purity chlorine dioxide is shown in Fig. S2. The detection method of the experimental sample is shown in Fig. S3; the small beaker box represents the solid chlorine dioxide sample, and the liquid in the bottle is the absorbent.

The effective $\mathrm{ClO}_{2}\left(G_{0}\right)$ was calculated using Eq. 1,

$$
\mathrm{ClO}_{2}(m g)=\left(\mathrm{V}-\mathrm{V}_{0}\right) \times C \times 13.49 \times 1000
$$

Where $\mathrm{V}$ is the volume of $\mathrm{NaS}_{2} \mathrm{O}_{3}$ standard solution consumed by samples $(\mathrm{mL}), \mathrm{V}_{0}$ is the volume of $\mathrm{NaS}_{2} \mathrm{O}_{3}$ standard solution consumed by blank $(\mathrm{mL}), \mathrm{C}$ is the concentration of $\mathrm{NaS}_{2} \mathrm{O}_{3}$ standard solution (0.001096 mol/L), $13.49 \mathrm{mg} \mathrm{ClO}_{2}$ is equivalent to $1 \mathrm{~mol} \mathrm{NaS}_{2} \mathrm{O}_{3}$.

\section{Definition of release characteristic parameters}

The release flux $F$ is the quality of $\mathrm{ClO}_{2}$ gas that is released by the BP-SCD sample in terms of unit-area and unit-time $\left(\mu \mathrm{g} \cdot \mathrm{h}^{-1} \cdot \mathrm{cm}^{-2}\right)$, as shown in Eq. 2,

$$
F=\frac{G}{t S} \quad\left[\mu \mathrm{g} \cdot \mathrm{h}^{-1} \cdot \mathrm{cm}^{-2}\right]
$$

where $S$ is the release area $\left(\mathrm{cm}^{2}\right), t$ is the release time (days), $G$ is the quality of released $\mathrm{ClO}_{2}$ gas at a certain time $(\mu \mathrm{g})$. The determination of $G$ used the Malonic acid-iodometry method (Wei $e t$ al. 2018). The release rate $P$ is the ratio of the released $\mathrm{ClO}_{2}$ gas quality $(G)$ at unit time and the initial quality $\left(G_{0}\right)$ of the effective $\mathrm{ClO}_{2}$ was calculated using Eq. 3,

$$
P=\frac{G}{G_{0}} \times 100 \% \quad[\%]
$$

where the total effective release time $\left(t_{r}\right)$ is the continuous release time when the sample started to release $\mathrm{ClO}_{2}$ gas to achieve critical release flux $F_{e}\left(5 \mu \mathrm{g} \cdot \mathrm{h}^{-1} \cdot \mathrm{cm}^{-2}\right)$, measured in days. Blank contrast experiments were done when testing the release flux $F$ due to samples becoming oxidized via oxygen in the air and potentially creating errors in the analytical results. To reduce errors, the release flux $F$ is an average of three separately measured values.

\section{Release process diffusion model}

The release system for $\mathrm{ClO}_{2}$ was placed in a cylinder, which had three terminals closed and one terminal opened, so that the open terminal participated in the mass transfer. The process could be simplified into a one-dimensional mass transfer system, as long as the $\mathrm{z}$ component was considered for the mass transfer. The $\mathrm{ClO}_{2}$ molecules were diffused through the pores (shown in Fig. 3) along a winding channel, which had a coefficient of "bending rate" $\tau$ used to describe the relationship between the actual length of the porous media channel and the length of the porous nominal route $\left(z_{2}\right.$ to $z_{1}$ ). It was impossible to estimate the values of $\tau$ via the parameters of the materials; however, the results from using similar materials in the past were compared to conduct a reasonable and reliable estimation. The release kinetic equation of BP-SCD was deduced based on Fick's first diffusion law. The relationship between the release flux and the released $\mathrm{ClO}_{2}$ concentration difference based on Fick's law could be expressed as Eq. 4,

$$
\frac{d G}{d t}=-D_{e} S\left(\frac{d C_{D}}{d z}\right)
$$

where $d G / d t$ is the diffusion flux of the $\mathrm{ClO}_{2}$ gas which goes vertically through the unit area at unit time $(\mu \mathrm{g} / \mathrm{d})$, called apparent diffusion flux, $S$ is the area of the diffusion surface $\left(\mathrm{cm}^{2}\right), d C_{D} / d z$ is the concentration gradient of $\mathrm{ClO}_{2}$ at the diffusion surface $\left(\mu \mathrm{g} \cdot \mathrm{mol}^{-1}\right)$, the negative sign indicated that $\mathrm{ClO}_{2}$ spread in the direction of the decreasing concentration or the quality of substance in the 
diffusion source decreased along with time; and $D_{e}$ is the effective diffusion coefficient which relates to $\varepsilon$ (porosity rate), $\tau$ (curving rate), and the substance properties of the diffusion system and temperature. Fick's first diffusion law is only applicable to stable diffusion which has a constant stable concentration gradient. But the release system's diffusion effect cannot always occur in a stable condition, due to the continual reaction, and the concentration of the diffusion surface will vary with space, time, and concentration gradient, which is also expressed as a function of space and time. It can be assumed that the time changing rate of the concentration gradient is related to the concentration gradient at that time, as shown in Eq. 5,

$$
\frac{d\left(d C_{D} / d z\right)}{d t}=\alpha \frac{d C_{D}}{d z}
$$

where $\alpha$ is the time constant for the concentration gradient. As $D_{e}$ is not a strict constant and is related to $C_{D}$ (diffusion surface concentration, $\mu \mathrm{g} \cdot \mathrm{cm}^{-2}$ ), and $n$ is the concentration effect index, $D_{e}$ is expressed in shown in Eq. 6,

$$
D_{e}=\frac{\varepsilon D_{0} C_{D}^{n}}{\tau} \quad n>0
$$

where $D_{0}$ is a constant related only to the substance properties of the diffusion system and temperature, used to determine the effective diffusion coefficient, called the intrinsic diffusion coefficient. When the initial condition of $t=0$, the initial concentration gradient of the diffusion surface $\left(d C_{D} / d z\right)_{t=0}=C_{0}\left(\mu \mathrm{g} \cdot \mathrm{mol}^{-1}\right)$. Therefore, Eq. 7 can be expressed as,

$$
\frac{d C_{D}}{d z}=C 0 e^{\alpha t}
$$

To calculate Eq. 8, Eq. 6 and Eq. 7 were substituted into Eq. 4:

$$
\frac{d G}{d t}=-\frac{\varepsilon}{\tau} D_{0} C_{D}^{n} S C_{0} e^{\alpha t}
$$

As the space is large enough to accept the $\mathrm{ClO}_{2}$ gas or the released $\mathrm{ClO}_{2}$ gas would migrate into another place, the change in $\mathrm{ClO}_{2}$ gas concentration in the environment to the $\mathrm{ClO}_{2}$ gas concentration at the release surface could be negligible. Therefore, the $\mathrm{ClO}_{2}$ gas concentration at the release surface at any time apparently relates to the quality of the effective $\mathrm{ClO}_{2}$ in the system at a certain time, which presents a mostly monotonically increasing relationship. For simple calculations, there was a linear relationship, as shown in Eq. 9,

$$
C_{D}=\gamma G_{L}
$$

where $\gamma$ is the concentration influence coefficient and $G_{L}$ is the quality of the remaining effective $\mathrm{ClO}_{2}$ in the solid sample (mg). To calculate Eq. 10, Eq. 9 was substituted into Eq. 8,

$$
\frac{d G}{d t}=-\frac{\varepsilon}{\tau} D_{0} \gamma^{n} G_{L}^{n} S C_{0} e^{\alpha t}
$$

Equation 10 is based on a modified Fick's first diffusion law, and was used to calculate the release kinetic equation for the BP-SCD slow-releasing $\mathrm{ClO}_{2}$, shown in Eq. 11,

$$
K_{D}=\frac{\varepsilon}{\tau} D_{0} \gamma^{n} S C_{0}
$$

which was used to calculate Eq. 12.

$$
\frac{d G}{d t}=-K_{D} e^{\alpha t} G_{L}^{n}
$$


Equation 12 is the release kinetic equation of the BP-SCD simplified using Eq. 10. The constant $K_{D}$ is called the intrinsic release constant which relates to the internal porosity rate, bending rate, intrinsic diffusion properties of the release material, temperature, the initial concentration of release surface, the release surface area, concentration impact coefficient and concentration impact index. The constant $\alpha$ is the concentration gradient constant which synthetically reflects the change in the release surface concentration gradient. When $\alpha \neq 0$ and $n=$ $0,1,2$, the relationship between the remaining quality of $G_{L}$ (effective $\mathrm{ClO}_{2}, \mathrm{mg}$ ) and the time $(t)$ can be solved by Eq. 12, as shown in Eq. 13, 14 and 15 below.

$$
\begin{aligned}
& n=0: G_{L}=G_{0}-\frac{K_{D}}{\alpha}\left(e^{\alpha t}-1\right) \\
& n=1: G_{L}=G_{0} e^{-\frac{K_{D}}{\alpha}\left(e^{\alpha t}-1\right)} \\
& n=2: G_{L}=G_{0} \frac{\alpha}{\alpha+G_{0} K_{D}\left(e^{\alpha t}-1\right)}
\end{aligned}
$$

The quality of the initial effective $\mathrm{ClO}_{2}$ is expressed in terms of $G_{0}=100 \%(\mathrm{mg})$ and the cumulative release rate is defined as $P=\left(G_{0}-G_{L}\right) \times 100 / G_{0}=\left(1-G_{L}\right) \%$, as expressed using Eq. 16, 17, and 18 below:

$$
\begin{aligned}
& n=0: \quad P=\frac{K_{D}}{\alpha}\left(e^{\alpha t}-1\right) \\
& n=1: \quad P=1-e^{-\frac{K_{D}}{\alpha}\left(e^{\alpha t}-1\right)} \\
& n=2: P=\frac{K_{D}\left(e^{\alpha t}-1\right)}{\alpha+K_{D}\left(e^{\alpha t}-1\right)}
\end{aligned}
$$

\section{RESULTS AND DISCUSSION}

\section{Release Mechanism Analysis for Long-Acting and Slow-Release $\mathrm{ClO}_{2}$}

A stable $\mathrm{ClO}_{2}$ matrix, a slow-release activator, and an agar cross-linking agent in an aqueous solution were formed into a colloidal superabsorbent resin (known as a gelling agent). In the internal colloid, the network resistance structure of the formed gelling agent and a cross-linking agent greatly slowed down the stable $\mathrm{ClO}_{2}$ and acidic activating agent combination and reaction rate. The generated $\mathrm{ClO}_{2}$ gas diffusion rate was also reduced via the network resistance structure. The network structure of the BP-SAR superabsorbent resin is shown in Fig. 1. 


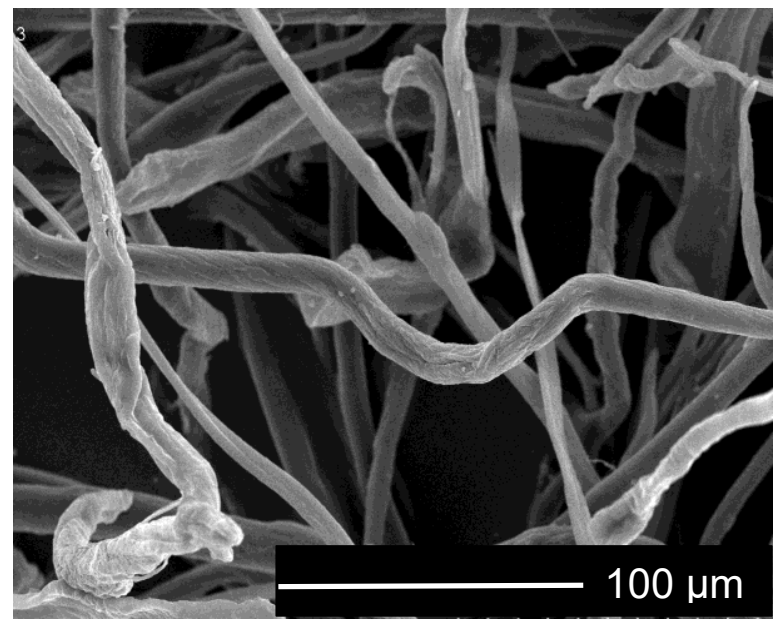

Fig. 1. SEM of BP-SAR $(\times 500)$

The curve of the release flux of the release system is shown in Fig. 2. BP-SCD is a longacting and slow-releasing solid release system, and its effective release time $\left(t_{r}\right)$ was up to 65 days. The $\mathrm{ClO}_{2}$ gas release flux was greater in the first few days and tended to stabilize after 10 days. This was due to the $\mathrm{ClO}_{2}$ gas diffusion force being larger during the initial stage, and the diffusion mainly occurred near the interface. The diffusion distance was small as well, so it showed a higher macro release flux during the initial stage. The diffusion rate of chlorine dioxide gas generated by the reaction was slowed down due to the impact of the BP-SCD mesh resistance structure. The entire release process exhibited diffusion control, which eventually leaded to a gradual decline in the release flux of macroscopic $\mathrm{ClO}_{2}$ gas. Due to the internal network structures, the diffusion resistance of the stable $\mathrm{ClO}_{2}$ molecules and the generated $\mathrm{ClO} 2$ gas molecules was substantially higher; the release process exhibited spreading control, leading to 134 the slow decline of the release flux of $\mathrm{ClO}_{2}$ gases macroscopically. This extended the $\mathrm{ClO}_{2}$ release time and achieved a slow release effect. With increasing time, the concentration difference became smaller inside and outside of the grid, which lead to a decreased diffusion force and diffusion distance. However, the resistance increased, which lead to the decline of the release flux. Due to the effect of the BP-SAR superabsorbent resin, the decline of the diffusion rate was slow, and the changing tendency of the macro release flux curve also showed a gentle state (as seen in Fig. 2). This characteristic could effectively control the $\mathrm{ClO}_{2}$ gas release flux and prolong service life for the release system. 


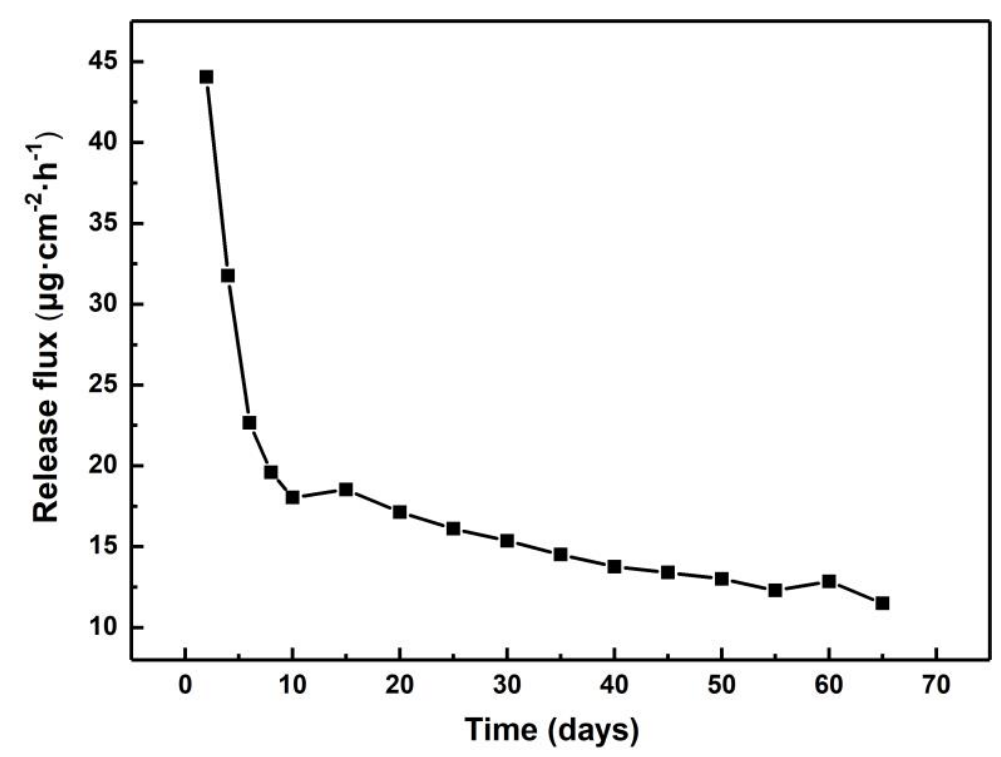

Fig. 2. Release flux of the solid release system for $\mathrm{ClO}_{2}$

\section{Release Process Analysis of Long-Acting and Slow-Releasing Solid $\mathrm{ClO}_{2}$}

The release process for $\mathrm{ClO}_{2}$ consisted of two stages: the diffusion control reaction stage and the $\mathrm{ClO}_{2}$ gas diffusion release stage. For convenient analysis, the release process was simplified, and the simplified model is shown in Fig. 3. In the diffusion control reaction stage, assume that stable $\mathrm{ClO}_{2}$ molecules $\left(\mathrm{ClO}_{2}^{-}\right)$and citric acid activator molecules $\left(\mathrm{H}^{+}\right)$were fully mixed into the solid. The main motivator was the concentration difference inside and outside of the grid (Fig. 3), which catalyzed the activation reaction, and because the network resistance structure slowed down the stable $\mathrm{ClO}_{2}$ and activator combination rate, the diffusion reaction stage became slower. Due to the large space in the solid sample being far larger than the size of the molecules, Fick's law can be used to describe how the molecules diffused in this space. The $\mathrm{ClO}_{2}$ gas diffusion and release stage occurred when the $\mathrm{ClO}_{2}$ matrix $\left(\mathrm{ClO}_{2}{ }^{-}\right)$and the acidic activating agent $\left(\mathrm{H}^{+}\right)$had an active reaction and produced $\mathrm{ClO}_{2}$ gas. Due to the slow rate of $\mathrm{ClO}_{2}$ gas production during the diffusion control reaction stage, as the $\mathrm{ClO}_{2}$ dissolved in the water and entered the pores of the solid samples; it formed a specific concentration difference, in which the $\mathrm{ClO}_{2}$ molecules spread to the outside space through the gap with the help of driving force from the concentration difference (Fig. 3). This process could be regarded as a single physical diffusion, and therefore could be described using Fick's diffusion law. 


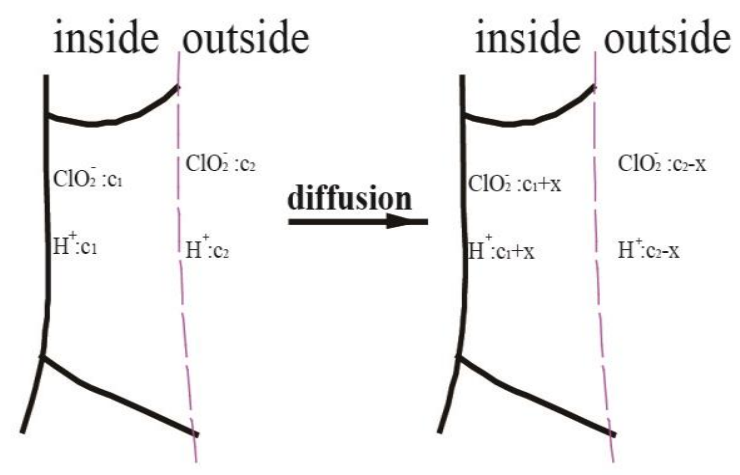

Diffusion control reaction stage

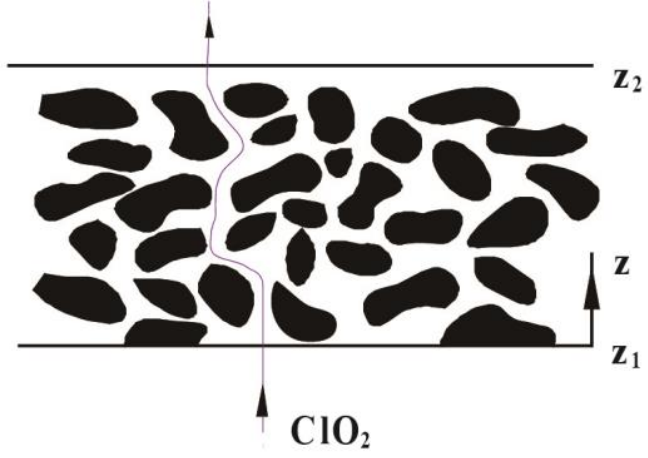

$\mathrm{ClO}_{2}$ gas diffusion release stage

Fig. 3. The $\mathrm{ClO}_{2}$ gas diffusion and release stage

\section{Verification and Evaluation of the Diffusion Model}

The BP-SCD sample was tested in a natural environment at room temperature $\left(25^{\circ} \mathrm{C}\right)$ and determined the quality $G_{i}(\mu \mathrm{g})$ of the released $\mathrm{ClO}_{2}$ at unit time, then calculated the data, termed $P$ (the cumulative release percentage of effective $\mathrm{ClO}_{2}(\%)$ shown in Table 1). Finally, the data were fitted to the new model calculated above, which compared the fitting results of a zero order kinetic model and a Higuchi model.

Table 1. Relationship of Cumulative Release Percentage and Time

\begin{tabular}{|c|c|c|c|c|c|c|c|c|}
\hline$t(\mathrm{~d})$ & 2 & 4 & 6 & 8 & 10 & 15 & 20 & 25 \\
\hline$P(\%)$ & 7.74 & 13.32 & 17.30 & 20.74 & 23.91 & 32.05 & 39.58 & 46.65 \\
\hline$t(\mathrm{~d})$ & 30 & 35 & 40 & 45 & 50 & 55 & 60 & 65 \\
\hline$P(\%)$ & 53.39 & 59.77 & 65.82 & 71.71 & 77.42 & 82.83 & 88.47 & 93.52 \\
\hline
\end{tabular}

$P$ is the $\mathrm{ClO}_{2}$ release rate and $t$ is time

The BP-SCD release curve was fitted with a kinetic model with Origin 8.0 software (OriginLab, Hampton, United States). As shown in Fig. 4, the relationship between the $\mathrm{ClO}_{2}$ release rate $(P)$ and time $(t)$ conformed to $P=k t+b\left(k>0, \mathrm{R}^{2}=0.9734\right)$; the linear relationship was strong. From a macroscopic perspective, the BP-SCD release process was in accordance with the apparent zero order kinetic model. The Higuchi model was a pore diffusion model (Higuchi 1961). As shown in Fig. 5, there was a strong linear relationship between the $\mathrm{ClO}_{2}$ release percentage and the square root of time, shown in $P=Q t^{1 / 2}\left(Q>0, R^{2}=0.9599\right)$, and the fitting results were better when the experimental data used the Higuchi model. The Higuchi model explained that the release behavior of the release system for $\mathrm{ClO}_{2}$ based on BP-SAR superabsorbent resin carrier better fit the Higuchi equation since BP-SAR had the characteristics of a porous control-release carrier. The relationship between the $\mathrm{ClO}_{2}$ release rate $(P)$ and the release time $(t)$ fit $P=\frac{K_{D}}{\alpha}\left(e^{\alpha t}-1\right)$ (when $n=0$ ), so the fitted curve was consistent with the experimental data (as shown in Fig. 6). Therefore, the three models can all explain the release rules and the characteristics of the slow-releasing $\mathrm{ClO}_{2}$ to a certain degree. 


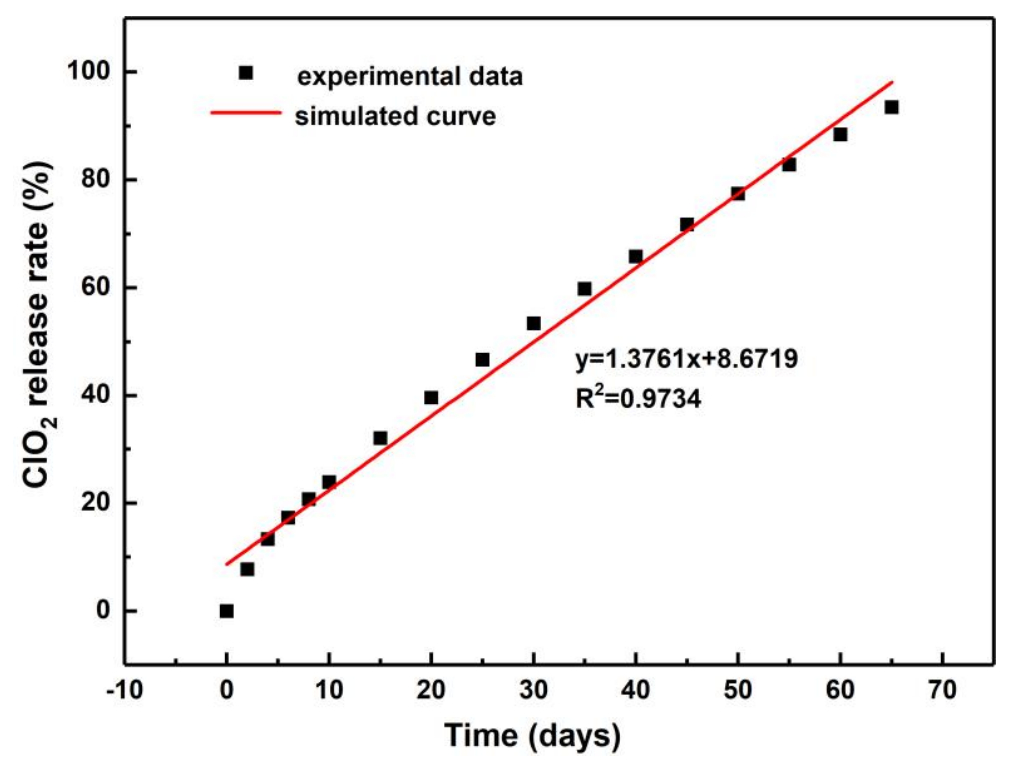

Fig. 4. Fitting results of the zero-order kinetic model

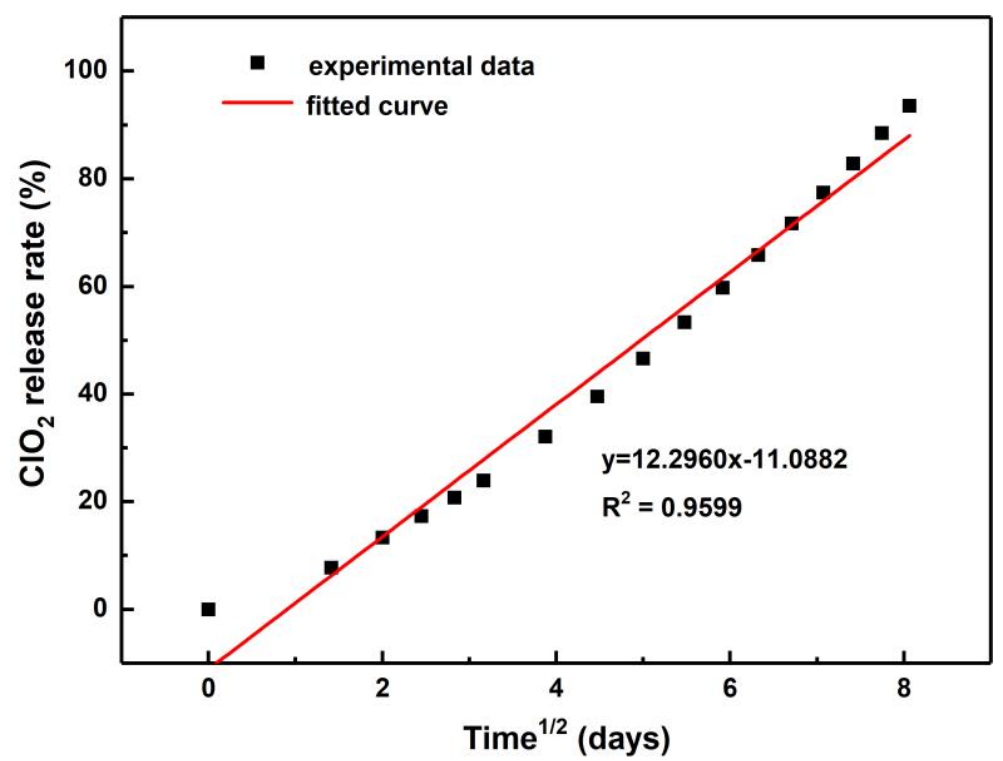

Fig. 5. Fitting results of the model 


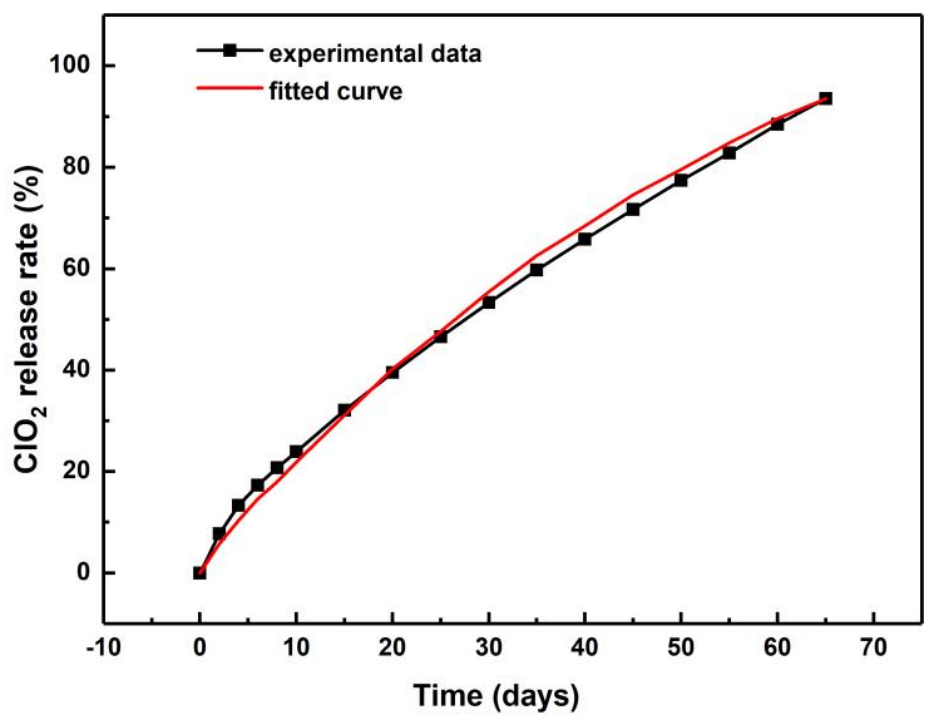

Fig. 6. Fitting results of the new kinetic model

The minimum Akaike information criterion (MAIC) (van Gastelen et al. 2017; Yamasaki et al. 2017) is a standard which measures the superiority of a model fitting, by estimating the complexity of the model and the superiority of the model fitting data. Therefore, according to the MAIC standard (van Gastelen et al. 2017) the better model from the three models which fit the release process characteristics can be selected and can also verify the reliability of the release kinetics models. The results are shown in Table 2, and the AIC formula is shown in Eq. 19,

$$
A I C=N \ln R S S+2 P=N \ln \left[\sum\left(F_{i}^{*}-F_{i}\right)^{2}\right]+2 P
$$

In this equation, $N$ is the number of test data, $P$ is the number of independent unknown parameters in the model, $R S S$ is the sum of the squared residuals, $F_{i}^{*}$ is the predicted value of diffusion rate in the model $\left(\mu \mathrm{g} \cdot \mathrm{h}^{-1} \cdot \mathrm{cm}^{-2}\right)$, and $F_{i}$ is the experimental measurement $\left(\mu \mathrm{g} \cdot \mathrm{h}^{-1} \cdot \mathrm{cm}^{-2}\right)$. As shown in Table 2 , the $A I C$ value is the minimum value for the new model established in this paper, which indicated the new model could reliably reflect the release features and rules of the release system for $\mathrm{ClO}_{2}$.

Table 2. AIC Values of Different Release Model for BP-SCD

\begin{tabular}{|c|c|c|c|}
\hline Model & Dynamics $(\mathrm{n}=0)$ & Higuchi & New $(\mathrm{n}=0)$ \\
\hline$A I C$ & 80.4599 & 90.5324 & 40.8534 \\
\hline
\end{tabular}

$A / C$ is the Akaike information criterion

Although the zero order kinetics model or the Higuchi model for BP-SCD could obtain a certain fitting effect, the new model based on a modified Fick's diffusion law achieved a better fit. The new model is different from the Higuchi model and Zero order kinetic model, since the newer model considered the concentration gradient changes as well as the time changes in the diffusion process. The initial estimated $K_{D}$ value was calculated via the linear least square method using the cumulative release rate and time data. The $\alpha$ value was estimated for the limit property in order to optimize the nonlinear least squares method, which led to the model parameters $K_{D}$ and an accurate estimate for the value of $\alpha$. The release kinetic equation for the release system for $\mathrm{ClO}_{2}$ was calculated using MATLAB 7.0 software (MathWorks, Natick, MA, USA) and shown in Eq. 20,

$$
P=-144.1534\left(\mathrm{e}^{-0.0161 \mathrm{t}}-1\right)
$$


where $K_{D}=2.3209$ and $\alpha=-0.0161$ were used for the optimization of the equation.

\section{Analysis of the New Model for the Release Process}

The long-acting and slow-releasing $\mathrm{ClO}_{2}$ products require a longer total release time $\left(t_{r}\right)$ at the effective concentration. When the total cumulative release rate $\left(P_{r}\right)$, calculated by Eq. 16, is close to being reached, the corresponding total release time $\left(t_{r}\right)$ is shown in Eq. 21 ,

$$
t_{r}=(1 / \alpha) \ln \left(1+\frac{\alpha}{K_{D}} P_{r}\right)
$$

Utilizing Eq. 11, the following measures extended the life of the $\mathrm{ClO}_{2}$ release system. First, to obtain a larger $t_{r}$ value, the total release percentage $\left(P_{r}\right)$ needs to be increased, ideally reaching $100 \%$, or as high as possible. Therefore, the amount of acidic active agent used should be adequate in the process design. When the chemical composition of system is constant, the release flux is constant as well, which allows the effective quality of $\mathrm{ClO}_{2}$ in the release system to be controlled, thus controlling the effective release time $\left(t_{r}\right)$. This also meant that the higher the chlorite levels are in the superabsorbent resin gel, the longer the release time and the longer period of validity for the deodorant and preservatives. Therefore, the ability to maintain and hold the liquid is very important to the superabsorbent resin and will effectively extend the life of the release system when prepared with a salt-tolerant resin that can absorb at greater magnifications and at a higher concentration of stable $\mathrm{ClO}_{2}$ liquid. If the effective release period for the $\mathrm{ClO}_{2}$ release system was extended to longer than six months, it would be widely used in the air sterilization, purification, and food preservation areas

Secondly, when the time changing constant $(\alpha)$ has a smaller concentration gradient, the concentration of the $\mathrm{ClO}_{2}$ matrix can be maintained for a longer time within a valid range, which extended the actuation duration and improved the overall effect. The smaller concentration gradient value can maintain the $\mathrm{ClO}_{2}$ macro-release rate at a low level for a long time. This property suggested that if the diffusion resistance of the stable $\mathrm{ClO}_{2}$ was artificially increased, possibly by adding a release agent (Zhou and Li 2006), it could avoid the burst release in the early stage and obtain a constant system speed, decrease the release flux and release quality at the initial release, as well as increasing the macro concentration of the stable $\mathrm{ClO}_{2}$ in the products in the later period, and increase the late diffusion impetus. This could effectively extend the $\mathrm{ClO}_{2}$ release time.

Because the total release time is related to the release surface area, it can achieve a slowrelease effect when the release area is reduced to under a certain release quality for the effective $\mathrm{ClO}_{2}$, i.e. designing products into a specific shape, such as barrel-shaped, the $\mathrm{ClO}_{2}$ can basically achieve a uniform release. When the porosity percentage was reduced, the bending rate was increased (Lee and Lee 2013). The $\mathrm{ClO}_{2}$ matrix dispersed or dissolved in the superabsorbent resin uniformly, then diffused and released through pores in the system. The properties of the carrier directly affect the controllability of the product, so future studies will include the network structure of the BP-SAR superabsorbent resin. This could achieve the controlled release of $\mathrm{ClO}_{2}$ via the changing of the structure of the carrier or adjusting the degree of cross-linking of the resin, to reach the purpose of a uniform slow-release.

From analysis of the new model, in order to get the purpose of long-acting and slowrelease, it can realize by several measures: prepare high salt tolerance resin which can absorb greater magnification and higher concentration of stable $\mathrm{ClO}_{2}$ solution; increased diffusion resistance of the stability of $\mathrm{ClO}_{2}$, such as changing the structure of high molecular polymer of carrier; adjusting the cross-linking degree of resin; adding a slow-release agent; reducing the release area; or designing a specific shape of product, such as barrel-shaped. 


\section{CONCLUSIONS}

1. A superabsorbent resin, made from bagasse pulp carboxymethyl cellulose for the slowrelease substrate and agar for the cross-linking agent, had a stable $\mathrm{ClO}_{2}$ solution (chlorite) and an acidic activator locked in the network of the resin to obtain a long-acting and slowreleasing solid product. The total effective release time for the product was 65 days, the preparation process is simple, the materials are easily degradable, the process is environment-friendly, and its cost is low.

2. The whole release process can be described using Fick's diffusion law, and the release kinetic equation was calculated as follows,

$$
\frac{d G}{d t}=-K_{D} e^{\alpha t} G_{L}^{n}\left(K_{D}=\frac{\varepsilon}{\tau} D_{0} \gamma^{n} S C_{0}\right)
$$

3. Compared to Zero order model and Higuchi model, the new model was advantageous for the filling of the data based on modified Fick's diffusion law. When $n=0$, the relationship between the $\mathrm{ClO}_{2}$ release rate percentage $(P)$ and release time is as follows: $P=\frac{K_{D}}{\alpha}\left(e^{\alpha t}-1\right)$

\section{AKNOWLEDGEMENTS}

This work was supported by the Guangxi Key Laboratory of Clean Pulp and Papermaking and Pollution Control (No. ZR201806-6); Construction Project of Characteristic Specialty and Teaching Base (Center) of Experimental Training of Undergraduate Universities in Guangxi from 2018 to 2020(T3050094101).

\section{REFERENCES CITED}

Ci, Y., Wang, L., Guo, Y., Sun, R., Wang, X., and Li, J. (2015). "Study on encapsulation of chlorine dioxide in gelatin microsphere for reducing release rate," International Journal of Clinical and Experimental Medicine 8(8), 12404-12410.

Fang, X. J., Chen, H. J., Gao, H. Y., Yang, H. L., Li, Y. L., Mao, P. C., and Jin, T. Z. (2016). "Effect of modified atmosphere packaging on microbial growth, quality and enzymatic defense of sanitizer washed fresh coriander," International Journal of Food Science \& Technology 51, 2654-2662. DOI: 10.1111/ijfs.13254

Han, J., Zhang, X., Liu, J., Zhu, X., and Gong, T. (2017). "Characterization of halogenated DBPs and identification of new DBPs trihalomethanols in chlorine dioxide treated drinking water with multiple extractions," Journal of Environmental Sciences 58, 83-92.

DOI: $10.1016 /$ j.jes.2017.04.026

Higuchi, T. (1961). "Rate of release of medicaments from ointment bases containing drugs in suspension," Journal of Pharmaceutical Sciences 50, 874-875. DOI:

doi.org/10.1002/jps.2600501018

Hsu, C. S., Lu, M. C., and Huang, D. J. (2015). "Disinfection of indoor air microorganisms in stack room of university library using gaseous chlorine dioxide," Environmental Monitoring and Assessment 187, 17. DOI: 10.1007/s10661-014-4235-2

Jiang, L., Feng, W., Li, F., Xu, J., Ma, Y., and Ma, H. (2015). "Effect of one-methylcyclo- 
propene (1-MCP) and chlorine dioxide ( $\mathrm{ClO} 2)$ on preservation of green walnut fruit and kernel traits," Journal of Food Science and Technology 52, 267-275. DOI: 10.1007/s13197013-0996-9

Kaur, D., Bhardwaj, N. K., and Lohchab, R. K. (2018). "A study on pulping of rice straw and impact of incorporation of chlorine dioxide during bleaching on pulp properties and effluents characteristics," Journal of Cleaner Production 170, 174-182.

DOI: $10.1016 /$ j.jclepro.2017.09.111

Lee, D. H., and Lee, O. (2013). "Control of generation of chlorine dioxide gas using polymer hydrogels containing acetic acid," Polymer Korea 37, 553-556. DOI: $10.7317 / \mathrm{pk} .2013 .37 .5 .553$

Long, M. X., Wang, H., Jiang, W., and Tan, S. M. (2016). "Antimicrobial activity of solid $\mathrm{ClO}_{2}$ preservative on miliang kiwifruit pathogenic fungi," Science \& Technology of Food Industry 37(4), 342-346. DOI: 10.13386/j.issn1002-0306.2016.04.060

Sun, X. X., Zhou, B., Luo, Y. G., Ference, C., Baldwin, E., Harrison, K., and Bai, J. H. (2017a). "Effect of controlled-release chlorine dioxide on the quality and safety of cherry/grape tomatoes," Food Control 82, 26-30. DOI: 10.1016/j.foodcont.2017.06.021

Sun, X. X., Baldwin, E., Ference, C., Narciso, J., Plotto, A., Ritenour, M., Harrison, K., Gangemi, D., and Bai, J. H. (2017b). "The effect of controlled-release chlorine dioxide on the preservation of grapefruit," HortScience 52(1), 122-126. DOI: 10.21273/HORTSCI11363-16

van Gastelen, S., Antunes-Fernandes, E. C., Hettinga, K. A., and Dijkstra, J. (2017).

"Relationships between methane emission of Holstein Friesian dairy cows and fatty acids, volatile metabolites and non-volatile metabolites in milk," Animal 11, 1539-1548. DOI: $10.1017 / \mathrm{S} 1751731117000295$

Wei, J., Chen, Y., Tiemur, A., Wang, J., and Wu, B. (2018). "Degradation of pesticide residues by gaseous chlorine dioxide on table grapes," Postharvest Biology and Technology 137, 142148. DOI: 10.1016/j.postharvbio.2017.12.001

Yamasaki, K., Fujisawa, A., and Nagashima, Y. (2017). "A proposal of Fourier-Bessel expansion with optimized ensembles of bases to analyse two dimensional image," Review of Scientific Instruments 88, 093507. DOI: 10.1063/1.5000744

Zhou, D. J., and Li, G. (2006). "Kinetics for release processes of the binary solid chlorine dioxide absorbed in carrier," Chemical Reaction Engineering \& Technology 22, 259-264. DOI: $10.3969 /$ j.issn.1001-7631.2006.03.012

Article submitted: May 27, 2018; Peer review completed: August 10, 2019; Revised version received and accepted: September 6, 2019; Published: September 19, 2019.

DOI: 10.15376/biores.14.4.8821-8834 


\section{APPENDIX}

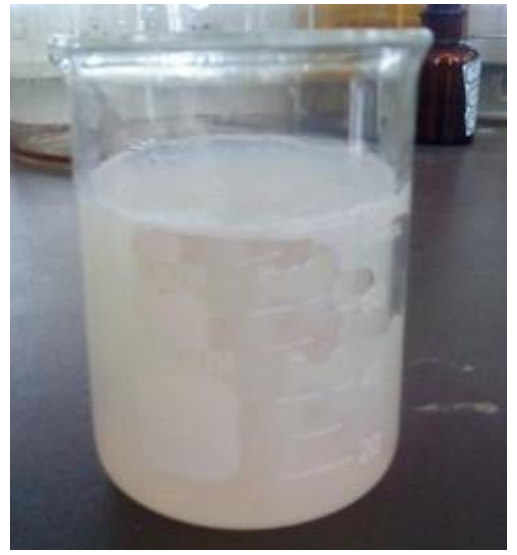

Fig. S1. Picture of the slow-releasing solid chlorine dioxide

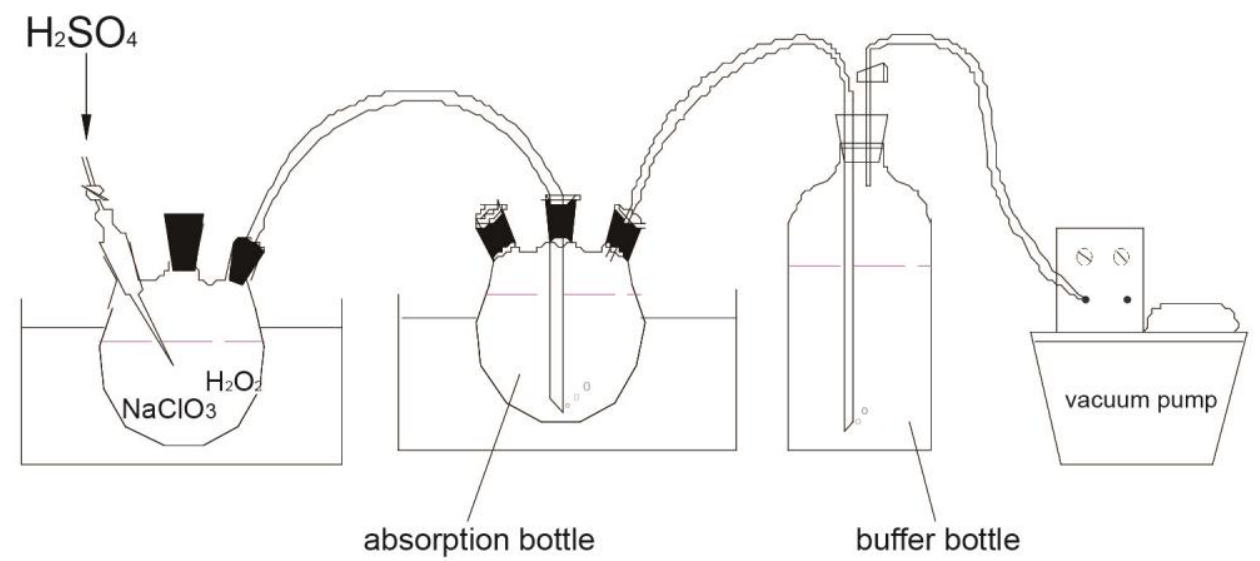

Fig. S2. Experimental device of preparing high-purity chlorine dioxide

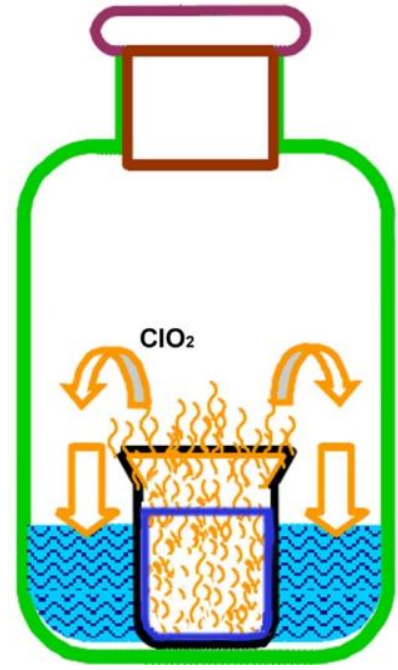

Fig. S3. Schematic diagram of determination of $\mathrm{ClO}_{2}$ releasing flux 